\title{
The use of curved vs. straight instruments in single port access surgery, on standardized box trainer tasks
}

\author{
Sanne Botden · Rob Strijkers • Sofie Fransen • \\ Laurents Stassen • Nicole Bouvy
}

Received: 4 May 2010/Accepted: 17 February 2011/Published online: 25 March 2011

(c) The Author(s) 2011. This article is published with open access at Springerlink.com

\begin{abstract}
Background Single-port access (SPA) surgery is a novel surgical technique to create nearly "scarless" surgery. SPA surgery appears to be safe and feasible, but the exposure and handling of tissue may not be optimal. Therefore, the performance of SPA surgery with different instruments used and conventional laparoscopy is compared.

Methods Fifteen participants ( $>50$ laparoscopic procedures) performed three basic tasks (translocation, clip \& cut, and tissue dissection, based on the fundamentals of laparoscopic surgery) in the box trainer in laparoscopy and SPA settings with both (conventional) crossed and curved instruments. All participants completed a questionnaire, which asked their opinion on the use of instruments and preference.

Results Translocation was performed significantly faster in both laparoscopy and SPA crossed than SPA curved (means, 130.3 and 137.7 vs. 170.7 sec; $p<0.001$ and $p=0.005$ ). The errors also were less in laparoscopy
\end{abstract}

S. Botden $(\varangle) \cdot$ R. Strijkers $\cdot$ S. Fransen $\cdot$ L. Stassen .

N. Bouvy

Department of Surgery, Maastricht University Medical Centre,

P. Debyeplein 25, Postal box 5800, 6202 AZ Maastricht,

The Netherlands

e-mail: Sanne_Botden@hotmail.com; S.Botden@mumc.nl

R. Strijkers

e-mail: Rob.Strijkers@gmail.com

S. Fransen

e-mail: Fransensofie@hotmail.com

L. Stassen

e-mail: Lps.stassen@mumc.nl

N. Bouvy

e-mail: N.Bouvy@mumc.nl and SPA crossed (means, 0.9 and 1.2 vs. 1.6), but not significant. The time to complete the dissection was almost equal between laparoscopy and SPA curved settings, but took longer for SPA crossed, although not significantly (148.1 and 150.8 vs. $179.5 \mathrm{sec}$ ). The errors only differed significantly between laparoscopy and SPA crossed (means, 0.5 vs. $1.27 ; p=0.044$ ). Fourteen participants still favored conventional laparoscopy and one SPA curved. They also thought SPA curved was better than crossed (means, 3.6 vs. $2.47 ; p=0.003)$ and that exposure is superior in curved (means, 3.4 vs. $2.27 ; p=0.002$ ).

Conclusions Although conventional laparoscopy may appear most effective for proper dissection and exposure of tissue, single-port access surgery shows potential. Especially in the tissue dissection task, there is no significant difference in time or errors between conventional laparoscopy and SPA surgery, using specially designed curved instruments. Although the participants favor conventional laparoscopy, this could evolve to a more accepting mind when SPA surgery becomes more available and used in the clinical setting.

Keywords Single port access surgery - Instruments . Laparoscopy

Laparoscopic cholecystectomy has been the "gold standard" for removal of a gallbladder since the early 1990s. This technique has gradually replaced the open cholecystectomy because of the shorter recovery time and hospital stay after the laparoscopic procedure compared with the open technique. Some drawbacks of this laparoscopic approach have been reported, such as pain from the subcostal incision and multiple scars on the abdominal skin [1]. 
To overcome this, a new approach has been introduced: single-port access (SPA) laparoscopic surgery. SPA cholecystectomy was first described by Navarra et al. where two trocars were introduced through a single umbilical incision [2]. It allowed the surgeon to perform a cholecystectomy through a single $2-\mathrm{cm}$ incision in the umbilicus, which leaves a nearly "scarless" result after the surgery. Several studies have shown preliminary results suggesting that SPA cholecystectomy is an effective and safe method [3-8].

Although many advantages are hypothesized, such as fewer scars, shorter healing time, less pain, less wound infections, and less incisional hernias, there are downsides to this approach. Many have reported difficulties in handling of the instruments and clashing of the camera while performing single-port surgery. Loss of triangulation makes dissection of Calot's triangle more difficult because of parallel entry of the working instruments. The use of a crossover setting makes it more difficult for the surgeon to operate because of interference of instruments with each other and the laparoscopic camera, and the counteracting movements of the instrument, compared with conventional laparoscopy.

The following suggestions are made to conquer these difficulties: different ports, curved instruments, and flexible endoscopes [4, 6, 9]. Several instruments have been developed for single-port access surgery, such as curved instruments, with a single or double curve; however, single-curved instruments still make a crossover setting necessary. The double-curved instruments do not crossover intra-abdominal because of both a curve extraperitoneal and intraperitoneal.

The main focus of this study is the advantages and disadvantages of the use different instruments-conventional instruments used in a crossed way or double-curved instruments-during single-port access surgery. Another aspect of this study is the difference in opinion and performance between SPA and conventional laparoscopy for basic skills.

\section{Methods}

\section{Subjects}

The participants were recruited at the Surgical Department of the Maastricht Medical University Centre, Maastricht, The Netherlands. Participants suitable for this research were surgeons or surgical residents who have performed more than 50 laparoscopic procedures in the clinical setting. In total, 15 participants completed the study and questionnaire.

\section{Equipment}

For the abstract environment of the abdomen, a standard box trainer (Endo Innovation, Hertogenbosch, The Netherlands) was used. The tasks were based on the existing Fundamentals for Laparoscopic Surgery tasks [10] and are described below. The LESS port (Olympus, Hamburg, Germany) was used to enter the instruments in the abdomen during the single-port access settings. For the conventional laparoscopy setting, three 5-12-mm trocars (Covidien, Dublin, Ireland) were used. The Endo-Eye laparoscopic camera (Olympus) was connected to a screen, which was placed at an ergonomic height and localization for the participants of the study. For both the laparoscopy and SPA-crossed setting, conventional straight instruments (Olympus) were used. For the SPA-curved setting, doublecurved instruments (Olympus) were used. The materials for the tasks were handmade by the researchers and were reusable to create a standardized situation.

\section{Settings}

The participants performed the three tasks with three different settings.

The first setting was the conventional laparoscopy setting, in which the following instruments were used: standard laparoscopic scissors, forceps, dissection clamp, and endoclip-applier. Three trocars were used in this setting, one trocar for the laparoscope and the other two trocars for the instruments, placed in ergonomic positions to perform the tasks.

The second setting was the single-port access setting with conventional (straight) laparoscopic instruments, which had to be used in a crossed position to perform the tasks (Fig. 1). The LESS port was used to insert the instruments, which had three gel-ports, one 12-mm port (for the laparoscope) and two 5-mm ports (for the working instruments).

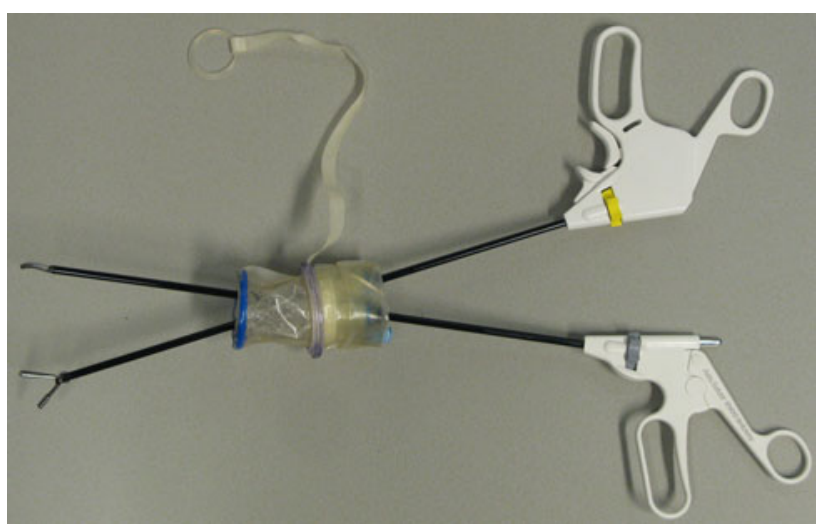

Fig. 1 Conventional (straight) laparoscopic instruments, used in a crossed way through a single port for SPA surgery 


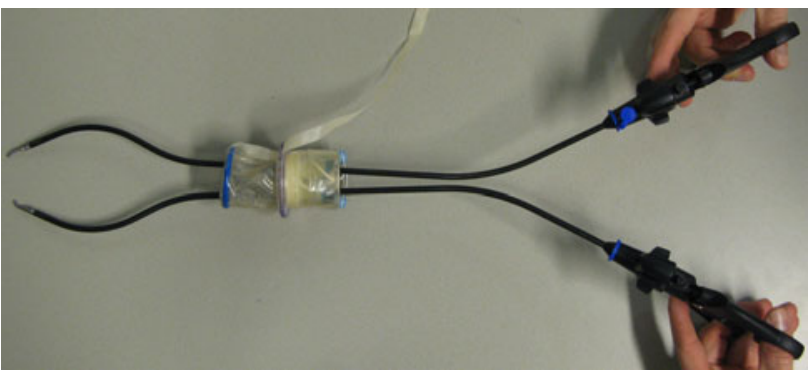

Fig. 2 Double-curved instruments, specifically designed for the use through a single port for SPA surgery

In the third setting, the LESS port also was used, but it differed from the second setting because the straight instruments were replaced with the double-curved alternatives (Fig. 2). The double-curved instruments available for this study were: scissors, forceps, and dissection clamp. There were no curved clip-appliers available; therefore, the clip and cut task could not be performed in this setting.

\section{Tasks}

The three tasks used in this study were based on the fundamentals of laparoscopic surgery (FLS), validated thoroughly validated training system for laparoscopic surgery [11]. They were specifically chosen to mimic the basic skills (such as dissection, exposure, and clip and cut) used in a laparoscopic cholecystectomy. The first task was designed to develop depth and visual-spatial perception in a monocular viewing system and the coordinated use of both the dominant and nondominant hand. The purpose of the second task was to replicate the placing of the clips and cuts during the division of the cystic duct and artery [11]. The third task was dissection to evaluate the possibility of dissecting Calot's triangle and to obtain the critical view of safety.

\section{Task 1: transfer rings}

A series of six iron rings had to be transferred from the right to the left pins on the tray in the box, by the right dissection clamp, and then transferred in the air to the left instrument and placed on the left side of the field. The participants had to transfer the rings from the left to the right pins in the same manner (Fig. 3). This exercise was scored by time of completion (with a cutoff time of $300 \mathrm{~s}$ ) and penalty scores: dropping of the ring and the inability to transfer a ring $[11,12]$.

\section{Task 2: clip application}

The participants had to place three hemostatic clips on a straw at premarked positions $3-\mathrm{cm}$ apart; following they had to cut on a mark halfway between the clips (Fig. 4).

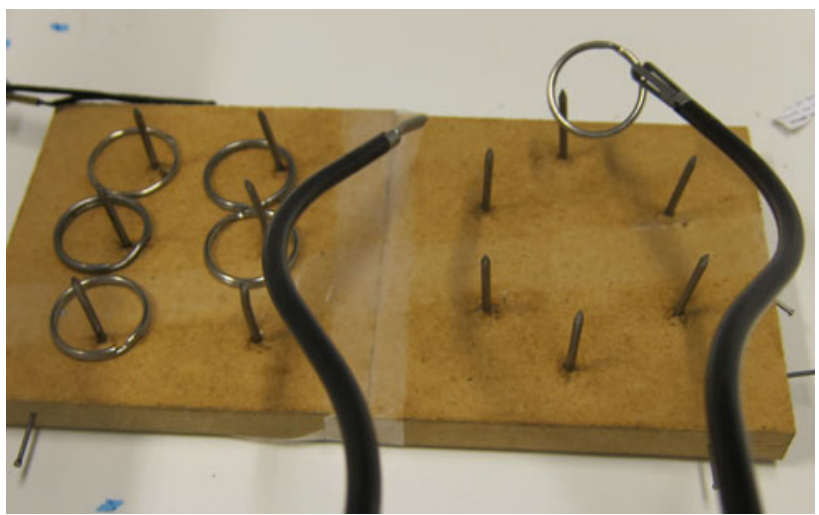

Fig. 3 Task 1, translocation of rings from one side to the other. In this image, the double-curved instruments are used

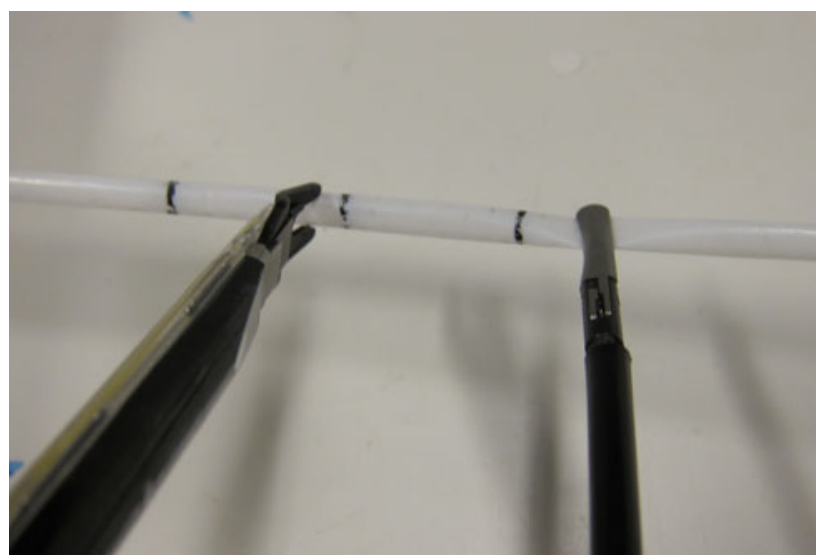

Fig. 4 Task 2, clip and cut of strings on the marked locations. In this image, the conventional (straight) instruments are used

The scores were determined by time of completion (with a cutoff time of $120 \mathrm{~s}$ ) and penalties. The penalty scores were calculated by measuring the deviation of the clip or cut from the predrawn lines and failure to place the clips properly on the tube. This task could not be performed in the SPA-curved setting, because there was no curved endoclip system available.

\section{Task 3: dissection}

The purpose of this task was to dissect a triangular shape out of a double-layer polyurethane glove. Only the upper layer of the double-layer glove had to be dissected and the second layer had to be preserved. The glove was placed at an ergonomic distance inside the box trainer at a $45^{\circ}$ angle (Fig. 5). A dissection clamp and scissors were used for this task. The scores were determined by time of completion (with a maximum of $300 \mathrm{~s}$ ) and penalties. The dissected part was analyzed and the margins from the drawn lines were measured to score the penalties; any damage to the second layer was taken into account. 


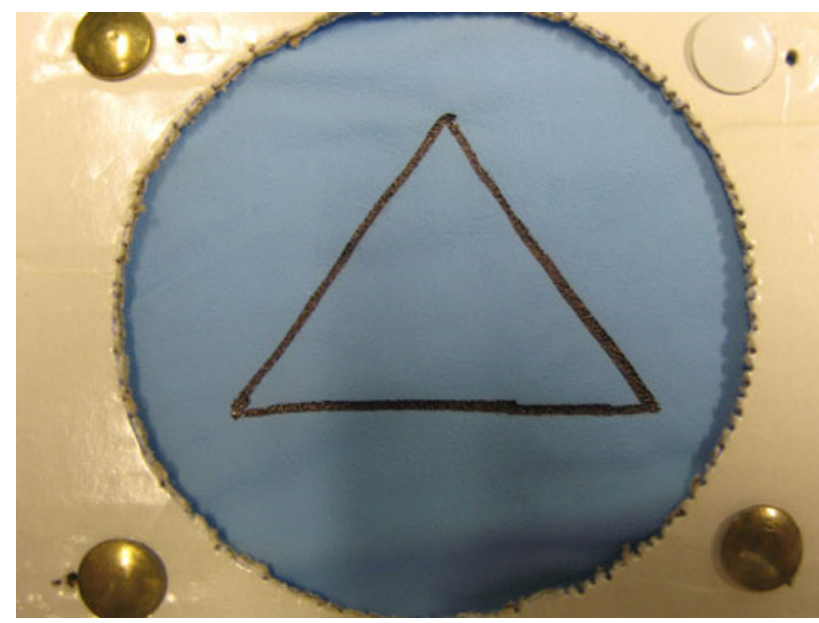

Fig. 5 Task 3, dissecting of a double layered glove, in which only upper layer of the marked triangle should be cut

\section{Protocol}

The 15 participants were randomly assigned to two groups. Both groups started with the conventional laparoscopy setting because they were all familiar with this surgical approach. Group A began the tasks in the SPA-crossed setting and next in the SPA-curved setting. Group B started the same tasks in the SPA-curved setting, followed by the SPA-crossed setting (Fig. 6). This construction was used to correct for a possible learning curve bias. All 15 participants completed the tasks on all three settings. In the conventional laparoscopy setting, the tasks were performed twice, whereas in the curved and crossed SPA setting, the tasks were performed three times. The second task was performed once in the conventional laparoscopy setting and twice in the crossed SPA setting because of the limited availability of endoclips and high cost of the disposable variant. The first runs of all the tasks in all settings were not considered in our results, to avoid the bias of unfamiliarity with both the task and the setting. In the SPA setting, both the second and third runs were processed to calculate the differences between the techniques and instruments. After the completion of the study, an evaluation form was filled out by the participants.

\section{Questionnaire}

The questionnaire, completed at the end of the study, consisted of three parts. The first part was about the
Fig. 6 The protocol of the study. The 15 participants were allotted to two groups to avoid bias in the results of the SPA settings

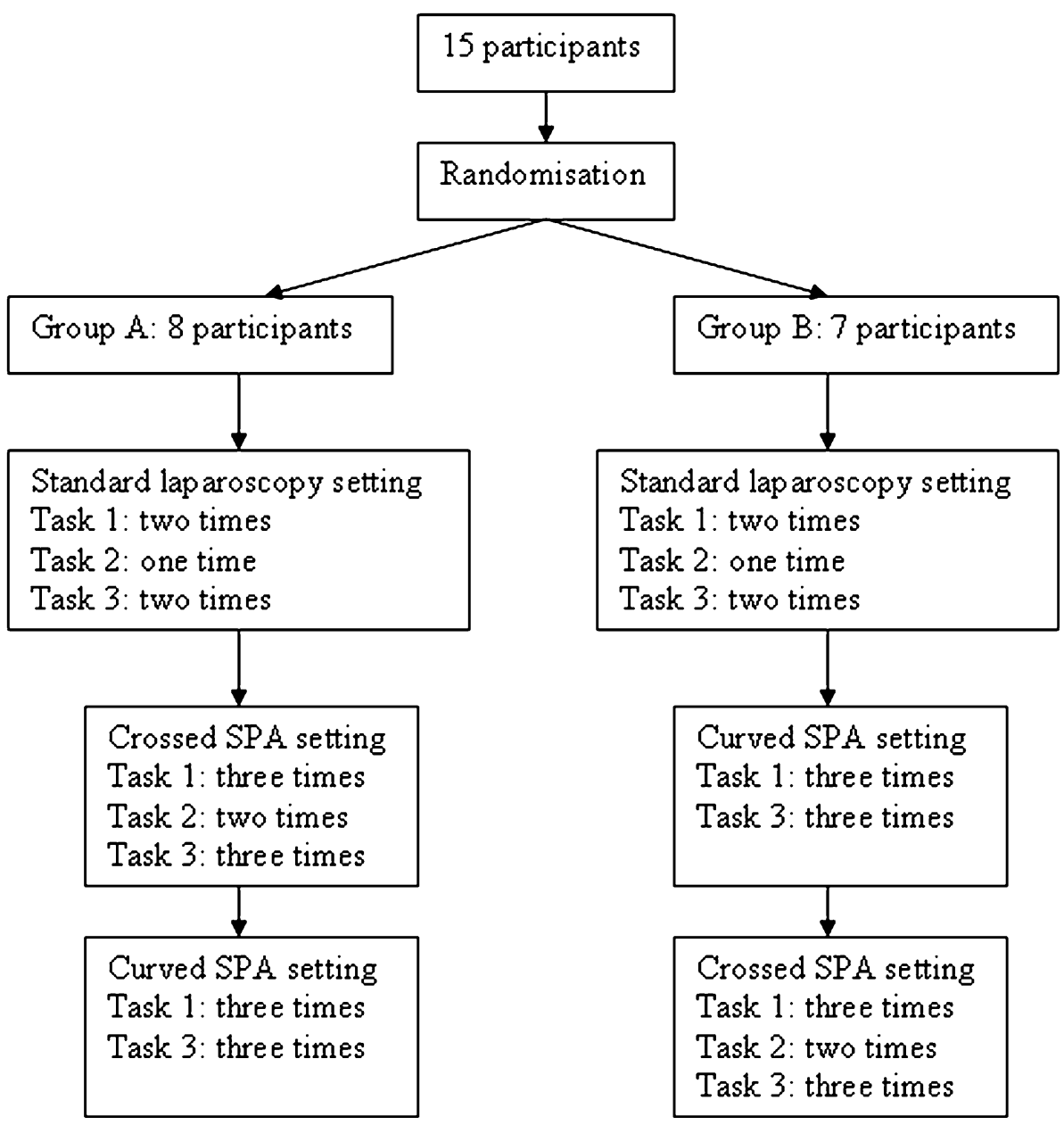


demographics and laparoscopic, SPA, and simulator experience of the trainees. In the second part, questions were asked regarding the handling and ergonomics of the instruments used in the different SPA settings (rated on a five-point Likert scale). The final questions were concerning the opinion and preference of the participants of the SPA setting versus the conventional laparoscopy setting. Any comments or recommendations at the end were recorded and processed for further evaluation. An informed consent was signed by all participants to state that they voluntarily participated in this study.

Data analysis

All data analysis was processed with Statistical Package for the Social Sciences $\left(\operatorname{SPSS}^{\circledR}\right)$ v 16.0. The scores "time" and "penalty scores" were measured for all tasks in all settings. All data of the laparoscopy tasks were used for the calculation of the differences. For the SPA setting, only the latter two tries were used. The first tries of tasks 1 and 3 were discarded in the statistical analysis to avoid bias in unfamiliarity. All data from the second task were taken into account during the calculations. The statistical differences between the performances on the tasks in the different settings were calculated with the paired $t$ test. The differences in opinion between the two SPA settings also were calculated with the paired $t$ test.

\section{Results}

\section{Demographics}

Two participants had performed SPA surgery before they entered the study, but this was both less than ten times. All participants had performed more than 50 laparoscopic procedures individually. There were eight surgical residents in the final years of training: five laparoscopic surgeons, one pediatric surgeon, and two general surgeons.

Crossed vs. curved instruments in SPA

The translocation of rings (task 1) was performed significantly faster in the SPA-crossed setting than the SPAcurved setting ( $p=0.005$ ), when comparing the third runs of the task with each other (Table 1). The penalty scores also were less; however, this was not a significant difference. The time to complete the dissection task (task 3) was almost $30 \mathrm{~s}$ shorter in the SPA-curved setting (in the third runs), but because of the wide standard deviation, this was not a significant difference. The errors were less in the SPA-curved setting, but again this was not significant (Table 1).

When comparing the second and third tries of the tasks in each setting, there was a significant difference between the second and third run of task 1 in the SPA-curved setting. The third try was performed significantly faster and

Table 1 Performance of the basic tasks in the laparoscopy, SPA-crossed, and SPA-curved settings

\begin{tabular}{|c|c|c|c|c|c|c|c|c|}
\hline \multirow{2}{*}{$\begin{array}{l}\text { Performance } \\
\text { on tasks }\end{array}$} & \multirow[t]{2}{*}{ Run } & \multirow[t]{2}{*}{ Score } & \multicolumn{3}{|c|}{ Mean (standard deviation) } & \multicolumn{3}{|l|}{$p$ value } \\
\hline & & & Laparoscopy & SPA crossed & SPA curved & $\begin{array}{l}\text { Laparoscopy vs. } \\
\text { SPA crossed }\end{array}$ & $\begin{array}{l}\text { Laparoscopy vs. } \\
\text { SPA curved }\end{array}$ & $\begin{array}{l}\text { SPA-crossed vs. } \\
\text { SPA curved }\end{array}$ \\
\hline \multirow[t]{4}{*}{ 1: Translocation } & \multirow[t]{2}{*}{2} & Time & $130.3(35.2)$ & $150.7(41.2)$ & $198.5(67.6)$ & 0.069 & $<0.001$ & 0.008 \\
\hline & & Errors & $0.87(0.83)$ & $0.80(1.15)$ & $2.33(1.68)$ & 0.855 & 0.012 & 0.004 \\
\hline & \multirow[t]{2}{*}{3} & Time & & $137.7(34.1)$ & $170.7(44.3)$ & $0.298^{\mathrm{a}}$ & $<0.001^{\mathrm{a}}$ & 0.005 \\
\hline & & Errors & & $1.20(1.66)$ & $1.60(1.24)$ & $0.541^{\mathrm{a}}$ & $0.102^{\mathrm{a}}$ & 0.472 \\
\hline \multirow[t]{4}{*}{ 2: Clip \& Cut } & \multirow[t]{2}{*}{1} & Time & $89.6(24.5)$ & & & & & \\
\hline & & Errors & $0.87(0.83)$ & & & & & \\
\hline & \multirow[t]{2}{*}{2} & Time & & $102.57(55.5)$ & & $0.398^{\mathrm{b}}$ & & \\
\hline & & Errors & & $1.73(2.15)$ & & $0.109^{\mathrm{b}}$ & & \\
\hline \multirow[t]{4}{*}{ 3: Tissue dissection } & \multirow[t]{2}{*}{2} & Time & $148.1(39.9)$ & $164.0(54.7)$ & $165.0(63.7)$ & 0.240 & 0.238 & 0.962 \\
\hline & & Errors & $0.53(0.74)$ & $1.13(1.12)$ & $1.27(0.59)$ & 0.108 & 0.001 & 0.685 \\
\hline & \multirow[t]{2}{*}{3} & Time & & $179.5(85.3)$ & $150.8(46.9)$ & $0.131^{\mathrm{a}}$ & $0.848^{\mathrm{a}}$ & 0.309 \\
\hline & & Errors & & $1.27(1.22)$ & 0.93 & $0.044^{\mathrm{a}}$ & $0.111^{\mathrm{a}}$ & 0.485 \\
\hline
\end{tabular}

All statistical differences are calculated with the paired $t$ test, a $p<0.05$ is considered a significant difference

a Statistical calculated difference between second run of the laparoscopy setting and third run of the SPA setting to account for the unfamiliarity with the SPA setting

b Statistical calculated difference between first run of the laparoscopy setting and second run of the SPA setting to account for the unfamiliarity with the SPA setting 
Table 2 The opinion of the participants on the instrument use in both SPA settings

\begin{tabular}{llll}
\hline Opinion on instruments & $\begin{array}{l}\text { Curved instruments } \\
\text { Mean (standard deviation) }\end{array}$ & Crossed instruments & \\
\hline Visualization of instruments & $3.47(0.83)$ & $3.13(0.92)$ & 0.313 \\
Angle of movement & $3.07(0.96)$ & $2.13(0.74)$ & $3.33(0.98)$ \\
Angle of entering abdomen & $2.87(0.92)$ & $2.40(1.06)$ & 0.025 \\
Ergonomic properties instruments & $2.80(0.86)$ & $2.27(0.59)$ & 0.204 \\
General opinion & $3.40(0.91)$ & $2.47(0.99)$ & 0.002 \\
Create a proper view of operating area & $3.60(0.83)$ & 0.003 \\
\hline
\end{tabular}

The statistical differences were calculated with the paired $t$ test. A $p<0.05$ is considered a statistically significant difference

Table 3 The general opinion on the use of the SPA settings during the tasks on the box trainer, rated on a five-point Likert scale

General opinion Mean (standard deviation)

Do you feel that the SPA surgery is a useful replacement for the laparoscopic cholecystectomy?

$2.3(0.9)$

Do you feel that the SPA setting was useful in the performance of task 1 ?

$2.9(1.03)$

Do you feel that the SPA setting was useful in the performance of task 2?

$2.9(1.1)$

Do you feel that the SPA setting was useful in the performance of task 3 ?

$3.0(1.36)$

Do you feel that the SPA-curved setting was more useful in the performance of task 1 than the SPA-crossed setting?

$3.0(1.07)$

Do you feel that the SPA-curved setting was more useful in the performance of task 3 than the SPA-crossed setting?

$3.9(1.16)$

with fewer errors than the second run $(p=0.05$ and $p=0.044)$. In the other task or setting, no significant differences between the performances were found within the tries of the tasks.

The participants were of the opinion that the SPAcurved setting was significantly better for the performance of these tasks than the SPA-crossed setting $(p=0.003)$. An important aspect of SPA surgery is the exposure of the operating field, which is rated significantly better in the SPA-curved setting as well $(p=0.002$; Table 2$)$. When asked about the preference of the instruments in the SPA setting, the participants were more likely to use the curved instruments (mean, 3.9; Table 3).

\section{Laparoscopy vs. SPA}

The first task (translocation of rings) was performed almost equal in the laparoscopy and SPA-crossed setting, when comparing the second run of the laparoscopy with the third run of the SPA-crossed setting (means, 130.3 vs. 137.7). However, when comparing the laparoscopy setting with the SPA-curved setting, for this task it was performed significantly slower in the SPA-curved setting $(p<0.001$, second run of laparoscopy vs. third run of SPA curved). The errors, however, do not differ significantly between these runs in these settings (Table 1). The second task (clip and cut) was only performed in the laparoscopy and SPA-crossed setting, for which no significant differences were found in the performance scores (Table 1). The time to complete the dissection task was almost equal for the second run of this task in the SPA-curved and -crossed setting, but in the third setting the time was improved in the SPA-curved setting but increased in the SPA-crossed setting. For the second run of this task in the laparoscopy setting and the third run in both SPA settings, the SPA-crossed setting took more than $30 \mathrm{~s}$ longer than the laparoscopy setting. However, this was not a significant difference because of the wide standard deviation. The errors on this task only differed significantly between the laparoscopy and SPA-curved setting for the second run but not in the third run. In the SPA-crossed setting, the errors also were increased in the third run, compared with the second run, which caused a significant difference with the laparoscopy setting $(p=0.044$; Table 1$)$.

When asked about the preference of the participants to perform a cholecystectomy, 14 still favored conventional laparoscopy and 1 the SPA-curved setting. The general opinion on the use of these instruments during the three tasks was quite neutral, and even negative when asked if it could be a proper replacement for conventional laparoscopic cholecystectomy (mean, 2.3; Table 3).

\section{Discussion}

Since the introduction of laparoscopy in the 1980s, surgeons all over the world are looking for less invasive ways to operate on patients. Single-port access surgery combines 
the cosmetic advantages with the technical advantage of normal laparoscopy. Through one umbilical incision, entry to the peritoneum is provided for all the instruments. Hiding the incision in the umbilicus makes this approach virtually scarless. NOTES (natural orifice transluminal endoscopic surgery) is a minimally invasive surgical method that uses natural orifices to gain access to the peritoneal cavity. Several studies have reported successful procedures, but closure of the visceral gap (mostly the gastric wall closure) is a problem, and therefore clinical use is not yet advisable [13-16]. It is easier for the surgeon to adapt to SPA, rather than NOTES, because the view and setup through the umbilicus is the same [17]. The cosmetic results of SPA are better than those of conventional laparoscopy. Surgeons should be aware that once SPA and NOTES are safe enough to perform, the public will demand these solutions.

It is suggested that SPA is possible with standard laparoscopic instruments, but there are a few downsides, such as interference of instruments with each other and the laparoscopic camera. The following suggestions are made to conquer these difficulties: different ports, curved instruments, and flexible endoscopes [4, 6, 9]. Chow et al. state that surgeons are currently operating with standard laparoscopic equipment, which is not designed for a singleincision approach [18]. The instruments developed for SPA surgery include the curved instruments, with a single or double curve. The single-curved instruments (also called reticulating instruments) are widely available but are not optimal because of the extraperitoneal interfering with the camera and each other. The double curved instruments do not crossover intra-abdominal because of both a curve extraperitoneal and intraperitoneal. This also should enhance the triangulation, and the second curving outside the body avoids clashing of the instruments and laparoscope extraperitoneal. To research the impact of the crossing over during SPA, double-curved and conventional laparoscopic (straight) instruments were compared in this study. The camera used in this study was a $5-\mathrm{mm}\left(30^{\circ}\right)$ Endo-Eye with coaxial light supply, in which extraabdominal interfering with the instruments should theoretically be avoided.

\section{Crossed vs. curved instruments in SPA}

Although the different instruments did not make a significant difference in the performance of the tasks, the question remains of whether there is a difference if used in the clinical setting. The translocation task was performed better in the crossed setting with the conventional instruments than in the SPA setting with the curved instruments. However, this can be attributed to the fact that this task could be performed with one instrument at a time; therefore, there was no clashing of the instruments and no real crossed setting. The dissection task was more difficult and might represent the clinical setting better, which showed a favorable outcome for the curved instruments. It seems that the most promising instruments for SPA surgery are doublecurved instruments, but there are still improvements that can be made. One of the comments made regularly by the participants was that the instruments were difficult to rotate when closed. This should be improved to create a better degree of motion. However, there was no correction for the fact that the surgeons were already familiar with the straight instruments and therefore did not have to adapt this matter, whereas in the curved setting, they had to adapt for both the setting and new instruments.

The participants were of the opinion that the curved instruments are more promising than the conventional instruments for SPA surgery; however, the ergonomics should be improved. There were some learning effects visible with the curved instruments; therefore, it is important that the surgeons train with these instruments before using them in the clinical setting. Follow-up studies should focus on the learning curves for the different kinds of instrument use in SPA surgery.

\section{Laparoscopy vs. SPA}

Although conventional laparoscopy may appear most effective for proper dissection and exposure of the tissue, SPA surgery shows potential. Especially in the tissue dissection task, there is no significant difference in time or errors between conventional laparoscopy and SPA surgery. However, the participants favor conventional laparoscopy, but this fact could evolve to a more accepting mind, when SPA surgery becomes more available and used in the clinical setting. It is important for more research to be performed on the best instruments and materials (SPA ports) to be used during SPA and that they will be improved where needed. Also, more research is needed on the clinical implementation of the SPA surgery and possible complications and disadvantages.

\section{Conclusions}

Although the translocation task is performed faster in both the laparoscopy and SPA setting (using crossed straight instruments), the dissection task (which is most representing for the clinical setting) is performed faster and with less errors with the curved instruments than in the crossed SPA setting, and equal to the laparoscopy setting. Participants still favor conventional laparoscopy for the clinical setting. When asked about the use of the instruments in the SPA setting, they preferred the use of curved instruments. 
Single-port access surgery can cause more challenges and difficulties to the surgeon during the operation; therefore, this study shows that the double-curved instruments show more potential for safe use than the crossed use of conventional instruments in single-port laparoscopic surgery.

Disclosures Dr. Sanne Botden, Rob Strijkers, Dr. Sofie Fransen, Dr. Laurents Stassen, and Dr. Nicole Bouvy have no conflicts of interest or financial ties to disclose.

Open Access This article is distributed under the terms of the Creative Commons Attribution Noncommercial License which permits any noncommercial use, distribution, and reproduction in any medium, provided the original author(s) and source are credited.

\section{References}

1. Keus F, de Jong JA, Gooszen HG, van Laarhoven CJ (2006) Laparoscopic versus open cholecystectomy for patients with symptomatic cholecystolithiasis. Cochrane database of systematic reviews (Online). (4):CD006231

2. Navarra G, Pozza E, Occhionorelli S, Carcoforo P, Donini I (1997) One-wound laparoscopic cholecystectomy. Br J Surg 84(5):695

3. Chow A, Purkayastha S, Aziz O, Paraskeva P (2010) Singleincision laparoscopic surgery for cholecystectomy: an evolving technique. Surg Endosc 24(3):709-714

4. Dutta S (2009) Early experience with single incision laparoscopic surgery: eliminating the scar from abdominal operations. J Pediatr Surg 44(9):1741-1745

5. Ersin S, Firat O, Sozbilen M (2010) Single-incision laparoscopic cholecystectomy: is it more than a challenge? Surg Endosc 24(1):68-71

6. Langwieler TE, Nimmesgern T, Back M (2009) Single-port access in laparoscopic cholecystectomy. Surg Endosc 23(5): $1138-1141$
7. Roberts KE, Solomon D, Duffy AJ, Bell RL (2010) Single-incision laparoscopic cholecystectomy: a surgeon's initial experience with 56 consecutive cases and a review of the literature. J Gastrointest Surg 14(3):506-510

8. Romanelli JR, Roshek TB 3rd, Lynn DC, Earle DB (2010) Single-port laparoscopic cholecystectomy: initial experience. Surg Endosc 24(6):1374-1379

9. Philipp SR, Miedema BW, Thaler K (2009) Single-incision laparoscopic cholecystectomy using conventional instruments: early experience in comparison with the gold standard. J Am Coll Surg 209(5):632-637

10. Aggarwal R, Moorthy K, Darzi A (2004) Laparoscopic skills training and assessment. Br J Surg 91(12):1549-1558

11. Derossis AM, Fried GM, Abrahamowicz M, Sigman HH, Barkun JS, Meakins JL (1998) Development of a model for training and evaluation of laparoscopic skills. Am J Surg 175(6):482-487

12. Fried GM, Feldman LS, Vassiliou MC, Fraser SA, Stanbridge D, Ghitulescu G, Andrew CG (2004) Proving the value of simulation in laparoscopic surgery. Ann Surg 240(3):518-525 discussion 525-528

13. Chamberlain RS, Sakpal SV (2008) A comprehensive review of single-incision laparoscopic surgery (SILS) and natural orifice transluminal endoscopic surgery (NOTES) techniques for cholecystectomy. J Gastrointest Surg 13(9):1733-1740

14. Pearl JP, Ponsky JL (2008) Natural orifice translumenal endoscopic surgery: a critical review. J Gastrointest Surg 12(7): $1293-1300$

15. Marescaux J, Dallemagne B, Perretta S, Wattiez A, Mutter D, Coumaros D (2007) Surgery without scars: report of transluminal cholecystectomy in a human being. Arch Surg 142(9):823-826 discussion 826-827

16. Rattner D (2006) Introduction to NOTES White Paper. Surg Endosc 20(2):185

17. Rao PP, Bhagwat SM, Rane A, Rao PP (2008) The feasibility of single port laparoscopic cholecystectomy: a pilot study of 20 cases. HPB (Oxford) 10(5):336-340

18. Chow A, Purkayastha S, Paraskeva P (2009) Appendicectomy and cholecystectomy using single-incision laparoscopic surgery (SILS): the first UK experience. Surg Innov 16(3):211-217 